\title{
Fault Diagnosis of Roller Bearing Based on PCA and Multi-class Support Vector Machine
}

\author{
Guifeng Jia, Shengfa Yuan*, and Chengwen Tang \\ College of Engineering, Huazhong Agricultural University, Wuhan 430070, P.R. China
}

\begin{abstract}
This paper discusses the fault features selection using principal component analysis and using multi-class support vector machine (MSVM) for bearing faults classification. The bearings vibration signal is obtained from experiment in accordance with the following conditions: normal bearing, bearing with inner race fault, bearing with outer race fault and bearings with balls fault. Statistical parameters of vibration signal such as mean, standard deviation, sample variance, kurtosis, skewness, etc, are processed with principal component analysis (PCA) for extracting the optimal features and reducing the dimension of original features. The multi-class classification algorithm of support vector machine (SVM), one against one strategy, is used for bearing multi-class fault diagnosis. The performance of the method proposed was high accurate and efficient.
\end{abstract}

Keywords: fault diagnosis, principal component analysis, features selection, multi-class support vector machine.

\section{Introduction}

Rolling bearing is one of the most widely used machinery components. It directly influences the operation of the whole machinery. Unexpected bearing failures can interrupt the production, cause unscheduled downtime and economic losses. So development of proper conditional monitoring and fault diagnosis procedure to prevent malfunctioning and failure of rolling bearings during operation is necessary. As a result, the fault diagnosis of rolling element bearings has been the subject of extensive research in recent years. To identify the most probable faults leading to failure, many methods are used for data collection, including vibration monitoring, thermal imaging, oil particle analysis, etc. Vibration analysis has been proved as the most effective and reliable method in diagnose. This article will apply the vibration signal to diagnose roller bearing faults. Then, the next step is signal processing.

Modern signal processing methods can be divided into two categories, one is the direct signal analysis or decomposition, such as short-time fast Fourier transform (STFT), wavelet transform, principal component analysis (PCA), blind source separation, which are mainly used on the signal basis processing or preprocessing, for instance, the time-frequency domain analysis, feature extraction, noise reduction and others. The other is the processing based on intelligence algorithms, such as artificial

${ }^{*}$ Corresponding author. Tel.: +86027 61268360.

E-mail: ysf@mail.hzau.edu.cn 
nerve networks (ANN), support vector machines (SVM), Bayes classification, decision tree, etc. The second class algorithms, artificial intelligence, are classifiers for fault diagnosis and decision. Two types of signal processing methods combined with organic is now the development trend of fault diagnosis field. This paper presented principal component analysis and multi-class support vector machines methods for signal processing and fault recognition.

The signal processing scheme as the follows;

(1) Calculating the parameters which discussed above for each sample to obtained an observed X.

(2) Selection principal features form the original parameters by principal component analysis method.

(3) Fault recognition, optimized samples would be training and classify applied multi-class SVM.

The procedure of experiment scheme was illustrated in fig.1.

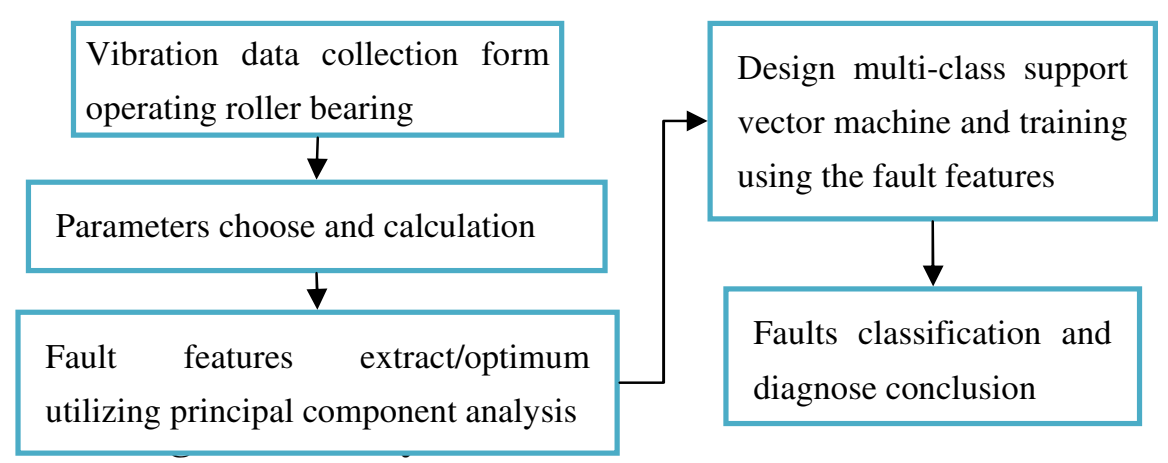

Fig. 1. Experiment scheme procedure

\subsection{Signal Data Parameters}

The formula 1-3 illustrated that frequency is principal parameters used in bearing diagnose.

$$
\begin{aligned}
\mathrm{f}_{b} & =\frac{1}{2} f\left[\left(1-\frac{d}{D}\right)^{2} \cos ^{2} a\right] \frac{D}{d} \\
\mathrm{f}_{i} & =\frac{1}{2} f\left[\left(1-\frac{d}{D} \cos a\right)\right] Z \\
\mathrm{f}_{o} & =\frac{1}{2} f\left[\left(1+\frac{d}{D} \cos a\right)\right] Z
\end{aligned}
$$

$\mathrm{fb}$, fi and fo are respectively outer race fault frequency, Inner race fault frequency, Balls spin frequency. Therefore, to accurately reflect the bearing fault type, it needs extract information form time domain and frequency domain. Consequently, the parameters 
applied in experiment were mean, standard deviation, sample variance, kurtosis, and skewness, peak-peak value and frequency [1]. Some complicated parameters and formulas were explained as following.

(1) Peak-peak value

$$
\mathrm{V}_{\mathrm{p}-\mathrm{p}}=\max \left(\mathrm{y}_{i}\right)-\min \left(\mathrm{y}_{i}\right)
$$

(2) Mean

$$
\mathrm{V}_{\mathrm{m}}=\frac{1}{N} \sum_{i=1}^{1} y_{i}
$$

(3) Standard deviation

Standard deviation is a measure of the efficient energy or power content of the vibration signal and clearly indicates deterioration in the bearing condition.

$$
\mathrm{SD}=\sqrt{\frac{n \sum_{i=1}^{n} y_{i}^{2}-\left(\sum_{i=1}^{n} y_{i}\right)^{2}}{n(n-1)}}
$$

(4) Sample variance

Sample variance is a measure of the amount of variation within the values of that variable, taking account of all possible values and their probabilities or weightings.

$$
\mathrm{SV}=\frac{n \sum_{i=1}^{n} y_{i}^{2}-\left(\sum_{i=1}^{n} y_{i}\right)^{2}}{n(n-1)}
$$

\section{(5) Kurtosis}

Kurtosis coefficient of the formation of substantial value that fault pulse the probability, kurtosis coefficient of the impulse response amplitude of the 4th power to determine the basis for improved signal to noise ratio, accuracy greatly enhanced when the bearing surface of the work of fatigue failure, The impact of face defect pulse, the greater the failure, the greater the impulse response amplitude, the more obvious faults.

$$
\text { Kurtosis }=\frac{\sum_{i=1}^{n}\left(\mathrm{y}_{\mathrm{i}}-\mu\right)^{4}}{\mathrm{~N} \sigma^{4}}
$$

\section{(6) Skewness}

Skewness is a measure of the degree of asymmetry of a distribution. If the left tail is more pronounced than the right tail, the function is said to have negative skewness. If the reverse is true, it has positive skewness. If the two are equal, it has zero skewness. 


$$
\text { Skewness }=\frac{1}{N} \sum_{i=1}^{\mathrm{n}}\left(\frac{y_{i}-\mu}{\sigma}\right)^{3}
$$

\section{(7) Frequency 1}

In the signal frequent domain, frequency is principal parameter for signal analysis, which contains the bearing fault frequent information. Frequency 1 was the frequency which was corresponds with the maximum amplitudes in power spectrum. Consequently, the vibration signal needed Fast Fourier Transform (FFT) in the first to obtain power spectrum.

\subsection{Principal Component Analysis}

Principal component analysis (PCA) involves a mathematical procedure that transforms a number of possibly correlated variables into a smaller number of uncorrelated variables called principal components. The first principal component accounts for as much of the variability in the data as possible, and each succeeding component accounts for as much of the remaining variability as possible. The performance of PCA is efficient for dimensionality reduction, which can reduce the complexity of signal processing [2] [3].

The article presented the PCA method for roll bearing vibration signal processing. The original statistical characteristics discussed in chapter 1.1.

The steps of PCA as follows:

(1) Standardize the sample observed matrix $X$ to get standardized $X^{*}$.

(2) Calculate the covariance matrix R.

(3) Calculate the eigenvectors and eigenvalues of the covariance matrix $R$.

(4) Choosing components and forming a feature vector.

(5) Deriving the new data set.

\subsection{Multi-class SVM Algorithms}

Support vector machine (SVM), an excellent algorithm used for classification and regression, was relatively new computational learning method based on the statistical learning theory, which developed by Vapnik. SVM classifier is better than ANN because of the principle of risk minimization. In ANN traditional empirical risk minimization was used on training data set to minimize the error. But in SVM, structural risk minimization (SRM) was used to minimize an upper bound on the expected risk. The SRM structure makes SVM good performance for reliable and accurate. Moreover, the algorithm sample requirement is small-scale. Therefore, SVM is very suitable for bearing fault diagnose. This article does not discuss the mathematical theory, it can be found in related books or papers conveniently. With the passage of time, SVM has been successfully applied to several filed such as detection, recognition, prediction, regression, fault diagnose, etc.

The original SVM algorithm is a binary classification where the class labels can take only two values: 1 and -1 , but in fact, the classify problems usually have more than two classes, take the bearing fault diagnose for example, there are inner race fault, outer 
race fault, balls fault and retainer fault [4]. Consequently, the multi-class SVM classification strategy will be utilizing for bearing multi-fault diagnoses. There have three strategy of multi-class SVM: one against one (OAO), one against all (OAA) and direct acyclic graph (DAG). This article applied OAO binary SVM to diagnose the fault types. One-against-one classifiers structure has $n *(n-1) / 2$ Classifiers and it employs the method of majority voting scheme to combine classifier and to evaluate classified result. Its principle was illustrated by formula 10-12 [5] [6] [7].

$$
\begin{aligned}
& \text { Minimize: } \frac{1}{2}\left(w^{i j}\right)^{T} w^{i j}+C \sum_{t} \xi_{t}^{i j} \\
& \text { Subject to: }\left(w^{i j}\right)^{T} \Phi\left(x_{t}\right)+b^{i j} \geq 1-\xi_{t}^{i j} \\
& \text { if } y_{t}=i\left(w^{i j}\right)^{T} \Phi\left(x_{t}\right)+b^{i j} \leq 1-\xi_{t}^{i j}, \\
& \text { if } y_{t}=j \quad \xi_{t}^{i j} \geq 0
\end{aligned}
$$

\section{Experiment Setup}

\subsection{Bearing Fault Data}

The time series vibration signals were obtained from the Case Western Reserve University Bearing Data Center for the following conditions: normal bearing, bearing with inner race fault, bearing with outer race fault and balls fault, and collected at 12,000 samples per second. The type of bearing used in experiment was 6205-2RS JEM SKF, deep groove ball bearing. The signal of each fault was displayed in fig. 2 .
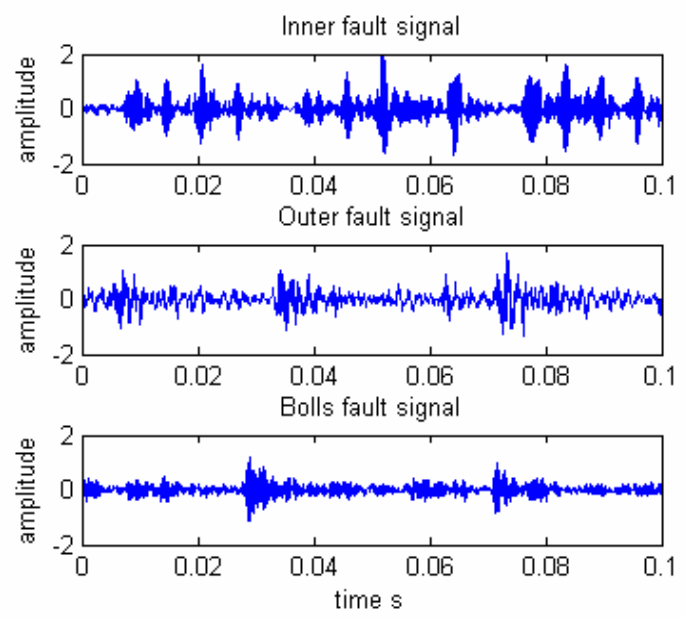

Fig. 2. Fault bearing signal 


\subsection{Features Selection Using Principal Component Analysis}

The original statistical features presented in chapter 1.1 were peak-peak value, mean, standard deviation, sample variance, skewness, kurtosis, frequency1. Experiment's scheme as follows: extract fifty samples from each fault signal and the length of each sample was 1,200 points, just 0.1 second. Consequently, it obtained 150 samples totally. Calculating the original parameters for each sample signal using the formula 4 to 9 achieve an observed matrix X. Then, processed observed matrix according to the steps of principal component analysis,

$$
X^{*}=\frac{X-X_{p}}{S}
$$

$\mathrm{X}^{*}$ stand for the standardized matrix, $\mathrm{Xp}$ was the mean of vibration signal and $\mathrm{S}$ was standard deviation. Firstly, the observed matrix X needs standardization according to formula 13.Then, calculated the correlation coefficient matrix $\mathrm{R}$ and its eigenvector, eigenvalues. The results of principal component analysis were presented in table 1 .

According to the table 1, it can learn that there were four principal components, named Prin1, Prin2, Prin3 and Prin4, selected from the original parameters. The Accumulative Contribution Ratio of principal components was 0.9883 , practically contained the information of original features.

Table 1. Principal component loading matrix L

\begin{tabular}{cllll}
\hline $\begin{array}{l}\text { Principal component } \\
\text { loading }\end{array}$ & Prin1 & Prin2 & Prin3 & Prin4 \\
\hline x1 & 0.4838 & -0.7661 & -0.4170 & 0.0424 \\
x2 & -0.9329 & -0.3373 & -0.0649 & -0.0407 \\
x3 & -0.9315 & -0.3276 & -0.0383 & 0.0883 \\
x4 & 0.8877 & -0.3105 & -0.3271 & 0.0035 \\
x5 & 0.0548 & 0.8350 & -0.5362 & -0.0346 \\
x6 & -0.9214 & -0.1459 & -0.2230 & -0.2597 \\
x7 & 0.9149 & -0.1680 & 0.2402 & -0.2368 \\
Contribution Ratio & 0.6356 & 0.2359 & 0.0974 & 0.0194 \\
Accumulative & 0.6356 & 0.8715 & 0.9688 & 0.9883 \\
Contribution Ratio & & & & \\
\hline
\end{tabular}

The last step was calculating ultimate result, principal component matrix Z, which was contained 98.8 percentage information of original parameters. Based on formula 14 , matrix $\mathrm{Z}$ was calculated by left multiplication loading matrix $\mathrm{L}$.

$$
\mathrm{Z}=\mathrm{X}^{*} * \mathrm{~L}
$$

Finally, principal component analysis was pre-processing stage for multi-class SVM. 


\subsection{Data Process and Fault Diagnose Utilizing Multi-class SVM}

Based on the characteristics of three bearing faults types, OAO strategic multi-class SVM algorithm was developed in this identification experiment. In this part, four parameters, selected by principal component analysis, were used for multi-class SVM, and every type fault contained 60 samples. Therefore, there were 180 samples used in training stage.

$$
\mathrm{Z}=\text { [Prin1 Prin2 Prin3 Prin4] T }
$$

Matrix $\mathrm{Z}$ was $180 \times 4$, contain the train information. Table 2 presented the setting of SVM training.

Table 2. Training parameters of MCSVM

\begin{tabular}{lc}
\hline Type of kernel functions & Liner \\
\hline Number of features used & 4 \\
Number of samples & 180 \\
\hline
\end{tabular}

Table 3 was the recognized result of multi-class SVM, which illustrated that the diagnostic correct accuracy of inner fault, outer fault and bolls fault separately were $85.7 \%, 98.9 \%$ and $96.9 \%$. The number of predicted samples was 294 and 276 samples recognized exactly. Correct accuracy in total was $97.3 \%$. The result showed that the OAO multi-class SVM was proper forbearing fault diagnose.

Table 3. Diagnosed result of MCSVM

\begin{tabular}{llll}
\hline Fault types & $\begin{array}{l}\text { Number of correct } \\
\text { classified } \\
\text { samples }\end{array}$ & $\begin{array}{l}\text { Number of incorrect } \\
\text { classified samples }\end{array}$ & $\begin{array}{l}\text { Predict } \\
\text { Accuracy }\end{array}$ \\
\hline Inner race fault & 98 & 14 & $85.7 \%$ \\
Outer race fault & 98 & 1 & $98.9 \%$ \\
Bolls fault & 98 & 3 & $96.9 \%$ \\
\hline
\end{tabular}

\section{Conclusions}

The paper presented the principal component analysis used in bearing fault features selection for reducing dimensions and the performance was efficient. As a result, obtained four principal components and its Accumulative Contribution Ratio nearly contain all original parameters information. Fault recognition using multi-class SVM, the correct ratio of classification totally is impressive. It is observed that the recognition performance of multi-class SVM technology is enhanced by PCA per-processing stage. 


\section{References}

1. Heng, R.B.W., Nor, M.J.M.: Statistical Analysis of Sound and Vibration Signals for Monitoring Rolling Element Bearing Condition. Applied Acrostics 53, 211-226 (1998)

2. Nimityongskul, S., Kammer, D.C.: Frequency domain model reduction based on principal component analysis. Mechanical Systems and Signal Processing 24, 41-51 (2010)

3. Serviere, C., Fabry, P.: Principal component analysis and blind source separation of modulated sources for electro-mechanical systems diagnostic. Mechanical Systems and Signal Processing 19, 1293-1311 (2005)

4. Sugumaran, V., Sabareesh, G.R., Ramachandran, K.I.: Fault diagnostics of roller bearing using kernel based neighborhood score multi-class support vector machine. Expert System With Application 34, 3090-3098 (2008)

5. Abbasiona, S., Rafsanjani, A., Farshidianfar, A., Irani, N.: Rolling element bearings multi-fault classification based on the wavelet denoising and support vector machine. Mechanical System and Signal Processing 21, 2933-2945 (2007)

6. Debnath, R., Takahidel, N., Takahashi, H.: A decision based one-against-one method for multi-class support vector machine. Pattern Analysis \& Applications 7, 164-175 (2004)

7. Manikandan, J., Venkataramani, B.: Study and evaluation of a multi-class SVM classifier using diminishing learning technique. Neurocomputing 73, 1676-1685 (2010) 